\title{
Supplementary information for Magnetic Vortex States in Toroidal Iron Oxide Nanoparticles: Combining Micromagnetics with Tomography
}

George R. Lewis, ${ }^{\dagger}, \|$ James C. Loudon, ${ }^{\dagger}$ Robert Tovey, ${ }^{\dagger}$ Yen-Hua Chen, ${ }^{\S}$ Andrew P. Roberts, ${ }^{*}$ Richard J. Harrison, "Paul A. Midgley, ' Emilie Ringe ${ }^{*, \dagger}, \|$

'Department of Materials Science and Metallurgy, University of Cambridge, Cambridge CB3 OFS, UK

"Department of Earth Sciences, University of Cambridge, Cambridge, CB2 3EQ, UK

Department of Applied Mathematics and Theoretical Physics, University of Cambridge, Cambridge, CB3 0WA, UK

${ }^{\S}$ Department of Earth Sciences, National Cheng Kung University, Tainan, Taiwan, 70101

${ }^{\#}$ Research School of Earth Sciences, The Australian National University, Canberra, Australia, ACT 2601

* Corresponding Author

\section{Contents}

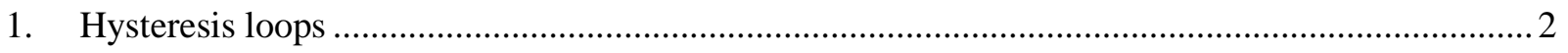

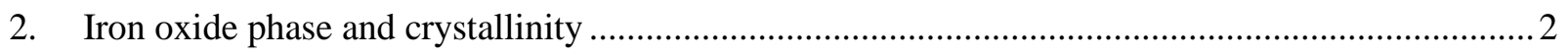

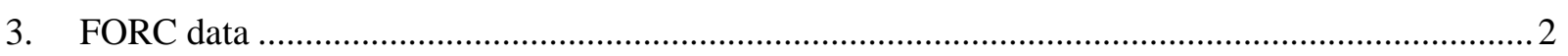

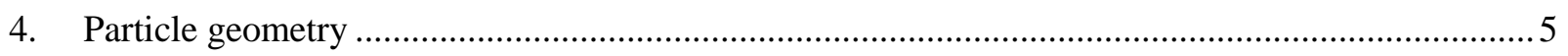

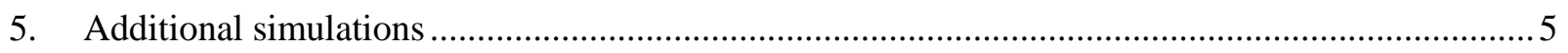

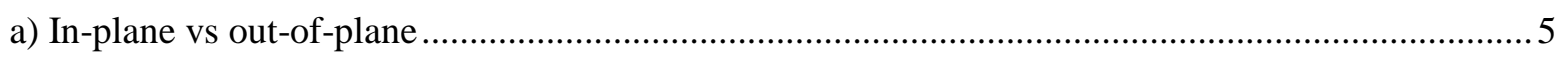

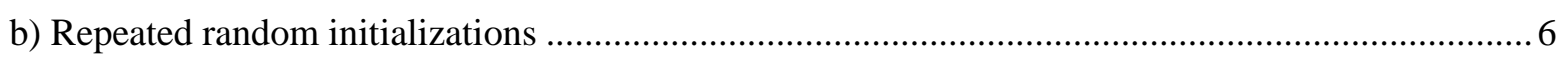

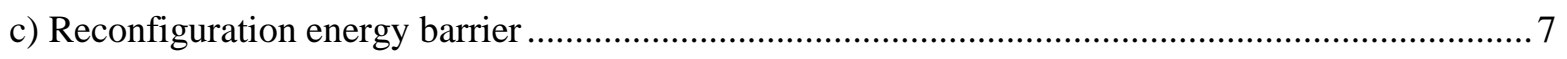

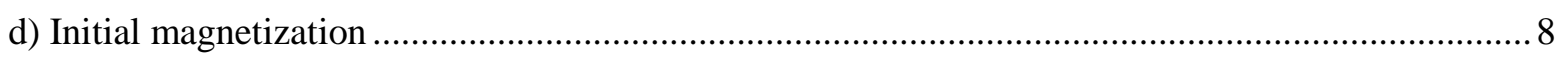

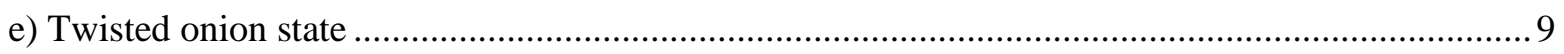

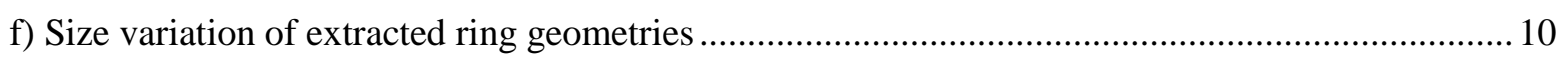

g) Onion state/vortex state transition pathway for real geometries.................................................. 11

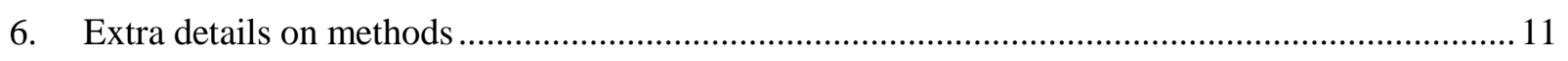

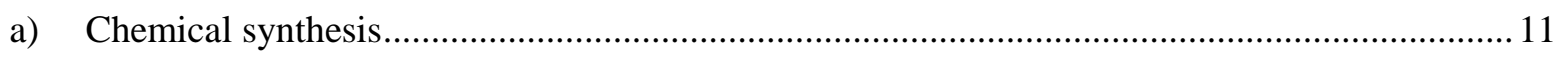

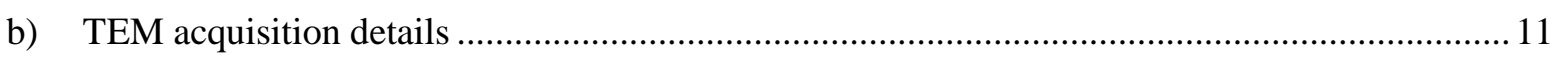

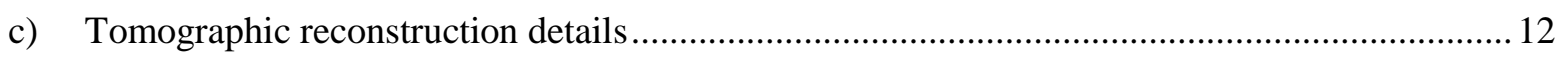

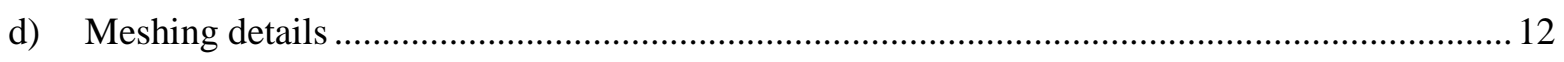

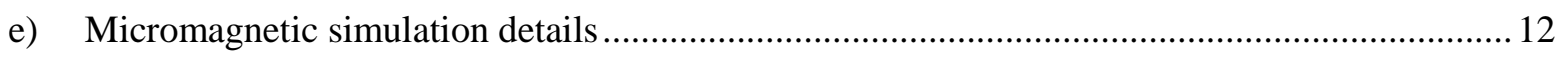

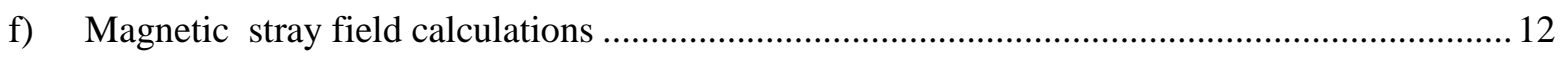

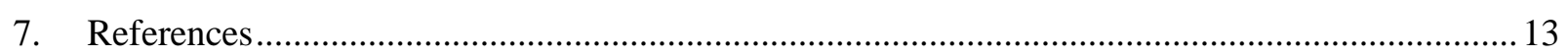




\section{Hysteresis loops}

Toroidal ferrimagnets are desirable due to their high saturation magnetization $M_{\mathrm{S}}$ (the asymptotic maximum magnetization $\boldsymbol{M}$ reached at high external magnetizing field $\boldsymbol{H}$ ) and their low remanent magnetization (the magnetization $\boldsymbol{M}$ remaining at zero applied field $\boldsymbol{H}$ ), as shown schematically in SI Figure S1. This allows particles to maintain stable dispersions, resistant to magnetic agglomeration (although particles should still be small enough to avoid gravitational sedimentation).

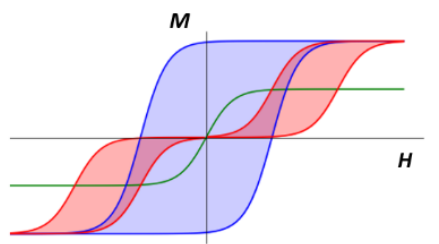

\begin{tabular}{|r|cc|}
\cline { 2 - 3 } \multicolumn{1}{c|}{} & Large $M_{\text {sat }}$ & Low $M_{\text {rem }}$ \\
\hline Standard ferromagnet & $\checkmark$ & $X$ \\
Superparamagnet & $X$ & $\checkmark$ \\
Toroidal ferromagnet & $\checkmark$ & $\checkmark$ \\
\hline
\end{tabular}

Figure S1 | Magnetic hysteresis in different nanomagnets. (Left) Sketch of magnetic hysteresis loops expected for standard ferromagnets, superparamagnets, and toroidal ferromagnets with (Right) saturation and remanence magnetization behavior of each. Note that the same trend would be expected whether the standard/toroidal samples are ferromagnetic or ferrimagnetic.

\section{Iron oxide phase and crystallinity}

There are 16 known iron oxide forms; the three most common are magnetite $\left(\mathrm{Fe}_{3} \mathrm{O}_{4}\right)$, maghemite $\left(\gamma-\mathrm{Fe}_{2} \mathrm{O}_{3}\right)$, and hematite $\left(\alpha-\mathrm{Fe}_{2} \mathrm{O}_{3}\right) ;^{1}$ these have reported saturation magnetization values of $92-100 \mathrm{Am}^{2} \mathrm{~kg}^{-1}$, 60-76 $\mathrm{Am}^{2} \mathrm{~kg}^{-1}$, and $0.3 \mathrm{Am}^{2} \mathrm{~kg}^{-1}$ respectively. ${ }^{2}$ Magnetite is the most strongly magnetic, so it is typically the oxide of choice for biomedical applications; however, magnetite eventually oxidizes to maghemite in the presence of atmospheric oxygen, ${ }^{3}$ so iron oxide samples often contain a mixture of magnetite and maghemite. Our micromagnetic simulations were made assuming magnetite nanoparticles because they have the most promising magnetic behavior. For maghemite particles, we expect the single-domain/vortex transition boundary to occur at larger particle sizes due to its lower saturation magnetization. Regardless of the oxide phase, the particle shape remains the same so its impact on the tomography component of this study is minimal.

Furthermore, Das et al. showed that multi-grain nanorings are unable to form vortex states at the same conditions as single-crystal nanorings. ${ }^{4}$ Our micromagnetic simulations assume single-crystal particles with the [111] axis oriented along $z$ as reported experimentally. ${ }^{5}$ However, grain boundaries and associated internal stresses influence magnetic domain formation in a complex way that makes it challenging to predict how our simulations would change for multi-grain nanorings. ${ }^{6}$

\section{FORC data}

The magnetic hysteresis behavior of both magnetite and maghemitized nanorings was measured using firstorder reversal curve (FORC) diagrams ${ }^{7,8}$ and also using the enhanced FORC protocol of Zhao et al. ${ }^{9}$ (SI Figure S2). The enhanced protocol enables the total FORC signal to be expressed as the sum of three separate FORC-like signals: the remanent FORC (remFORC), transient FORC (tFORC) and induced FORC (iFORC) components. For detailed explanations of the patterns observed in these signals see Roberts et al. ${ }^{10}$ and Harrison et al. ${ }^{11}$ The maghemitized sample has identical patterns of signal to the fresh magnetite sample, albeit with a reduced coercivity. The total FORC signal (a) has a broadly spread pattern typical of vortex state samples. The remanence FORC (b) contains a large broad central peak (labelled 1). There is a small peak at the origin (2) consistent with the presence of superparamagnetic (SP) particles with relaxation times far shorter than the measurement time (of the order $100 \mathrm{~ms}$ ) and an elongated signal (3) consistent with the presence of SP particles with relaxation times several times greater than the measurement time. The 
transient FORC (c) has two peaks (labelled 1 and 2) that are commonly associated with the internal-field driven nucleation of vortices. The induced FORC (d) shows evidence of both the negative-positive-negative (N-P-N) pattern associated with single-domain behavior and the negative-positive-negative-positive (N-P$\mathrm{N}-\mathrm{P})$ pattern associated with vortex behavior. ${ }^{10}$ These results suggest that the sample contains a mixture of predominantly vortex states with minor SP and SD contributions. The broad spread of intensities observed in the remanent and transient signals indicate a correspondingly broad spread of vortex nucleation and annihilation fields. 
(a) (Top) Fresh magnetite nanorings

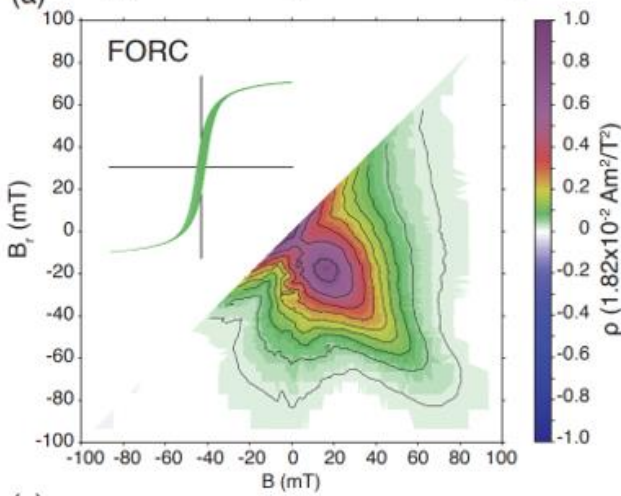

(c)
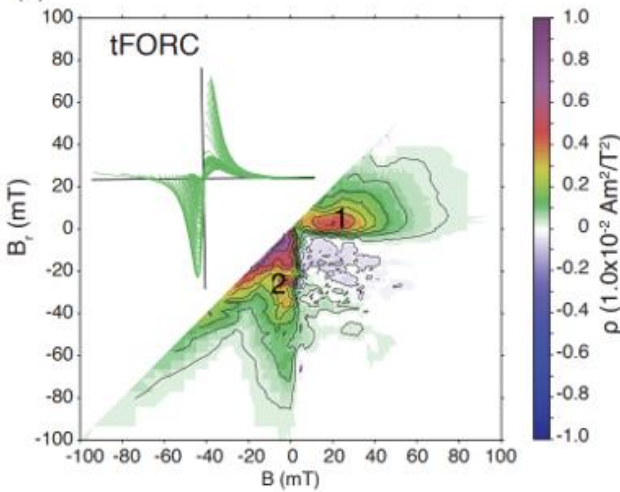

(a) (Bottom) Maghemitized nanorings
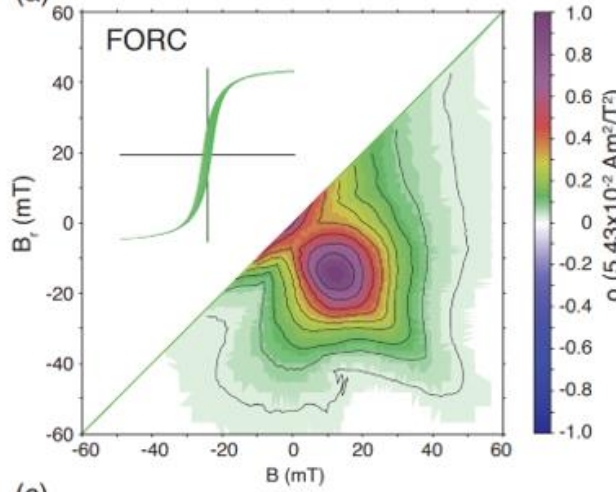

(c)

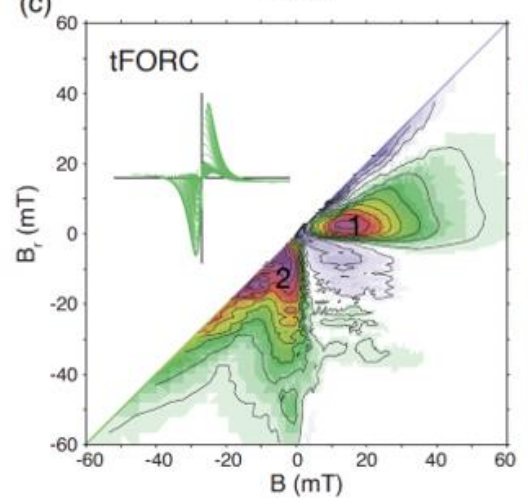

(b)

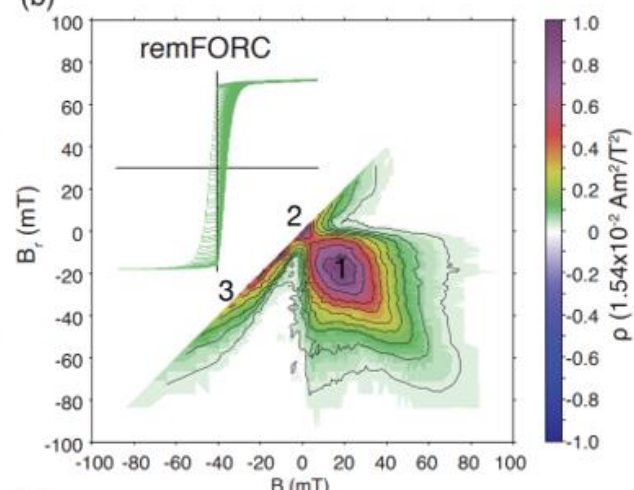

(d)

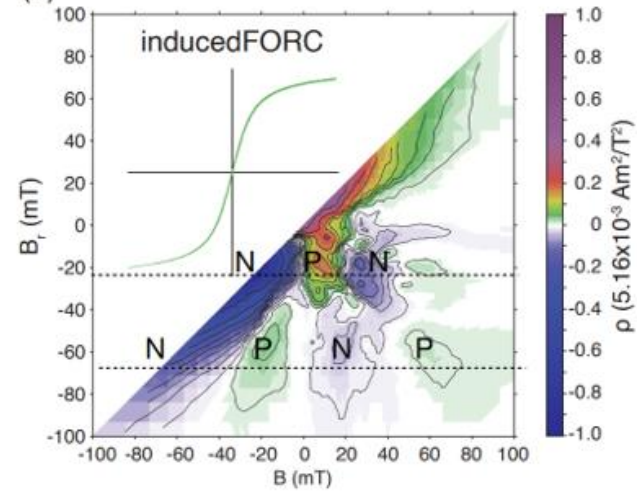

(b)
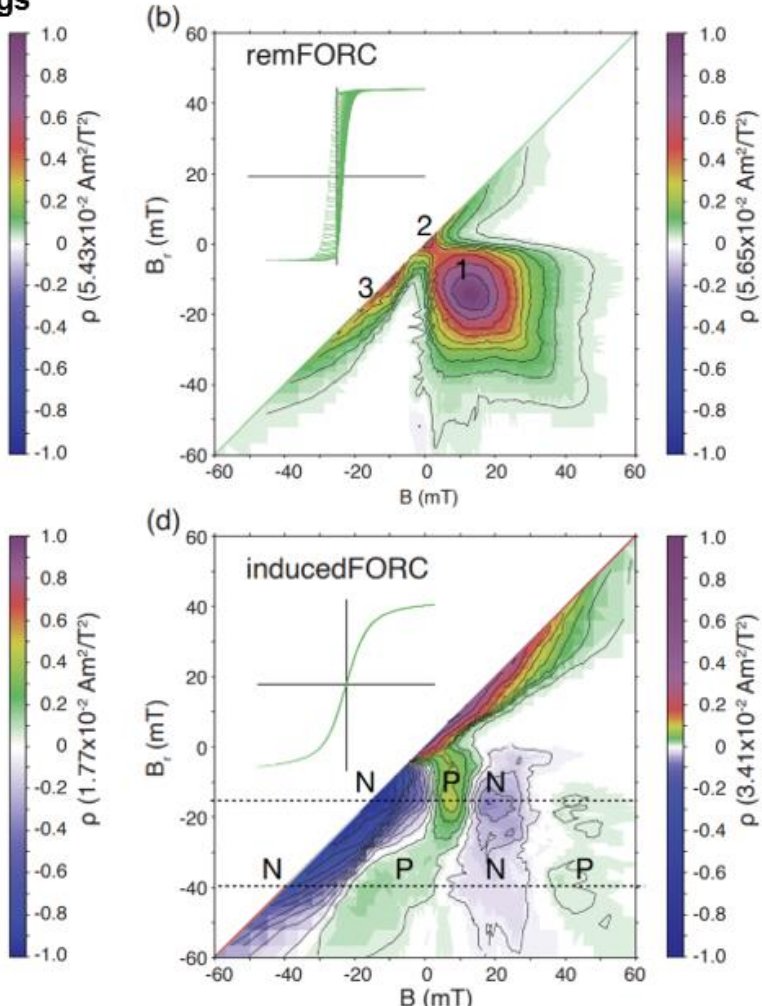

(d)

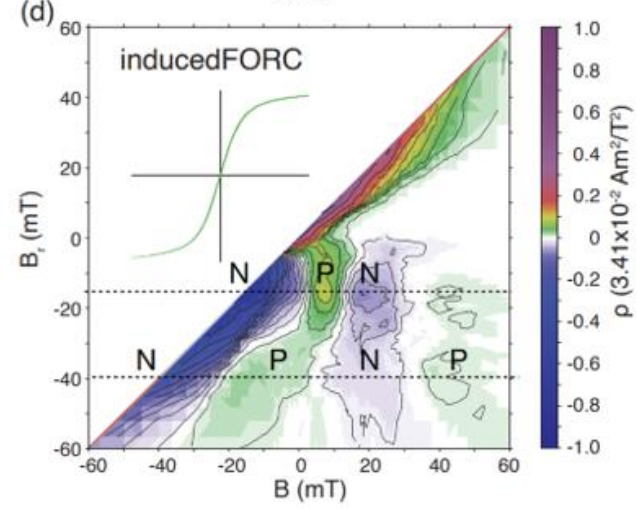

Figure S2 1 FORC analysis. Magnetic FORC data for (top) magnetite and (bottom) maghemite nanorings, showing the total FORC signal and its separated remanent, transient, and induced components. 


\section{Particle geometry}

The simulated particles were created as surface meshes in the open-source 3D graphics suite Blender using the parametric equations

$$
\begin{gathered}
x=(R+r \cos v) \cos u, \\
y=(R+r \cos v) \sin u, \\
z=\Gamma \sin v,
\end{gathered}
$$

[Equation S1]

where $x, y$ and $z$ are coordinates in space; $u$ and $v$ are parameters that vary between 0 and $2 \pi$; and $R, r$, and $\Gamma$ are geometric constants defined in SI Figure S3. Elliptical eccentricity $e$ is subsequently derived from these geometric constants,

$$
e=\sqrt{1-\frac{r^{2}}{\Gamma^{2}}}
$$
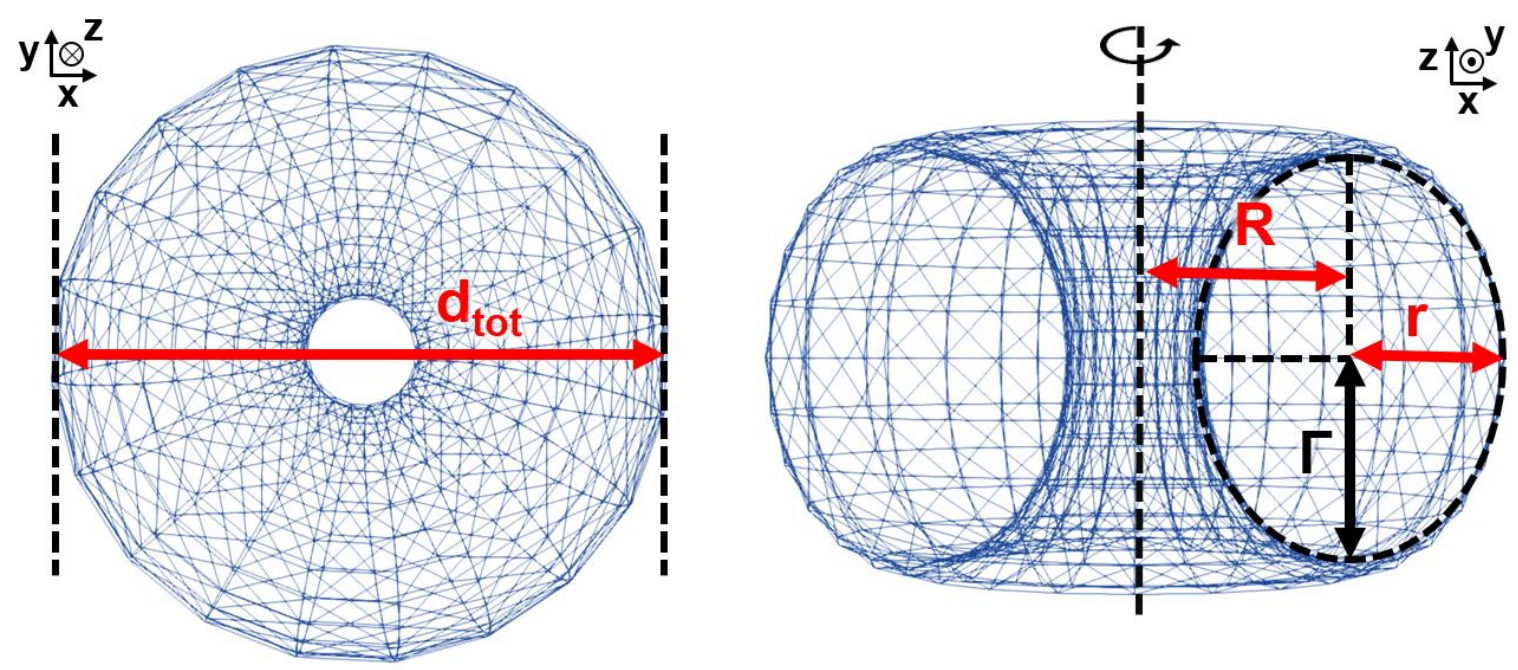

Figure S3 | Toroid geometry. Diagram illustrating the geometry and orientation of a model toroid, with parameters labelled that correspond to ring thickness $r / R$, total ring diameter $d_{\text {tot }}$, and elliptical eccentricity $e$.

Note that using a toroidal geometry avoids the sharp corners that are present in models that approximate the shape to hollow cylinders and instead model a shape more similar to the experimentally observed particles. This is likely to make a significant difference to the resulting magnetic behavior due to the tendency of magnetic fields to diverge from corners and their potential to act as pinning sites. ${ }^{12,13}$

\section{Additional simulations}

a) In-plane vs out-of-plane

The net magnetization magnitude $M / M_{\mathrm{S}}$ does not distinguish between single domains that are magnetized in-plane and those that are out-of-plane, so a further investigation was carried out to address this. The remanent magnetization of all toroids with $d_{\text {tot }}=50 \mathrm{~nm}$ was visualized; in-plane magnetizations are generally preferred by ring-like toroids with low eccentricity and out-of-plane magnetizations preferred by more tubular toroids with high eccentricity (SI Figure S4). Furthermore, a gradual transition between the two states is observed at high eccentricity when changing from thin to thick rings, which can be seen for 
the top left toroid in Figure S4 $(e=0.9, r / R=0.2)$, which is magnetized at an intermediate angle. We note that the out-of-plane state can be reached as a metastable configuration even for low eccentricity particles.

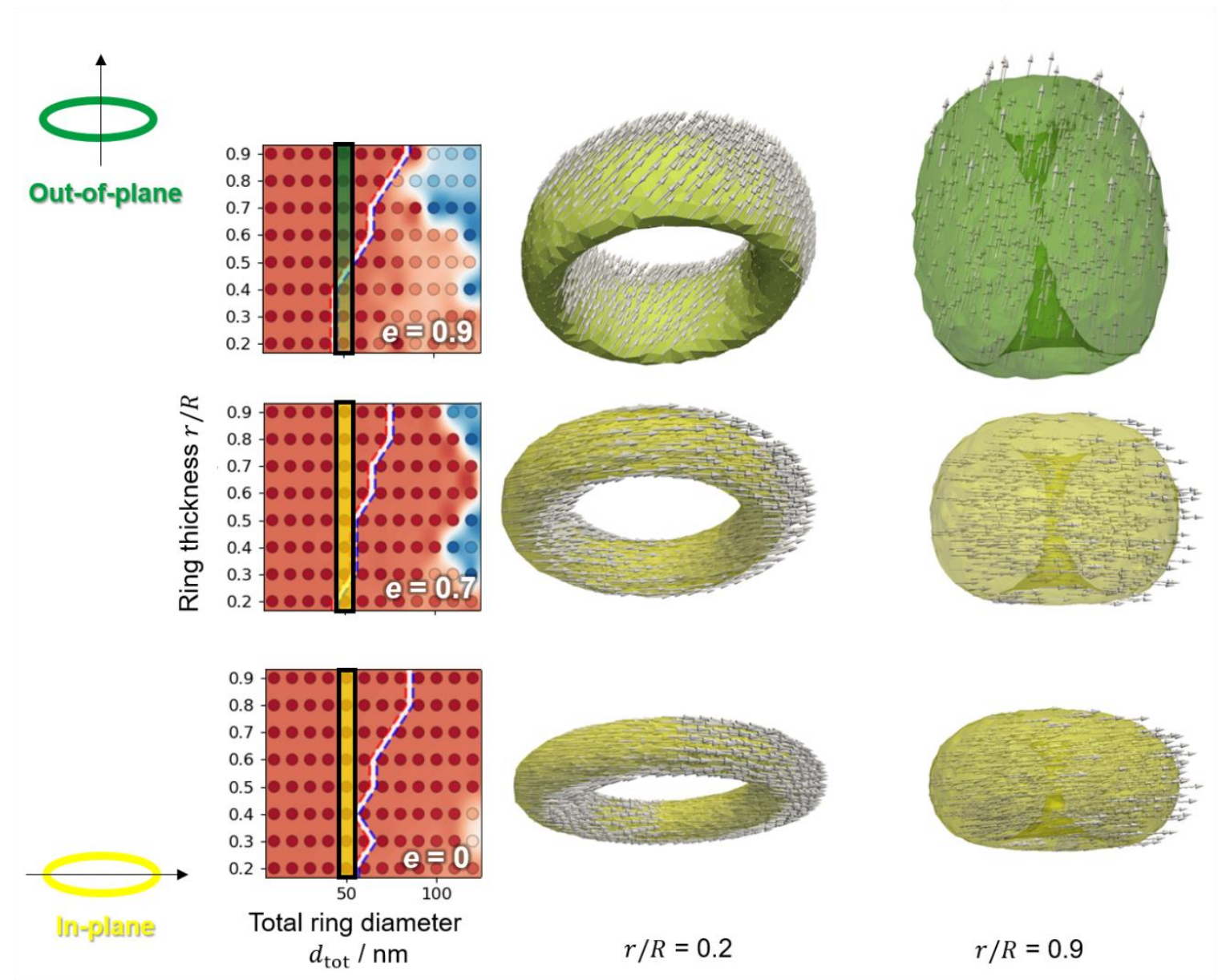

Figure S4 | In-plane vs out-of-plane magnetization. (Left) Portion of the phase diagram from Figure 2 with the regions analysed $\left(d_{\text {tot }}=50 \mathrm{~nm}\right)$ highlighted by boxes: yellow indicates an in-plane state and green indicates an out-of-plane state. (Right) Remanent magnetizations corresponding to $r / R=0.2$ and $r / R=0.9$ for each eccentricity.

This result agrees with other micromagnetic studies, ${ }^{14-16}$ where out-of-plane magnetizations become dominant for longer cylinders, which is similar to the effect of increasing eccentricity. This trend exists because toroids have shape anisotropy that encourages the easy axis to point along the most elongated direction. ${ }^{17}$

The distinction between the remanent state having in-plane and out-of-plane magnetization is important when looking at magnetic reversal behavior. However, for biomedical applications where the vortex remanent state is desired, both in-plane and out-of-plane states are unfavorable because they have a large stray field that produces less stable dispersions.

b) Repeated random initializations

For a typical toroid geometry $\left(e=0.7, r / R=0.4, d_{\mathrm{tot}}=70 \mathrm{~nm}\right)$, we calculated the remanent micromagnetic states from 1000 random initializations. The results indicate three possible states: vortex, onion (in-plane), and out-of-plane magnetization (SI Figure S5). 

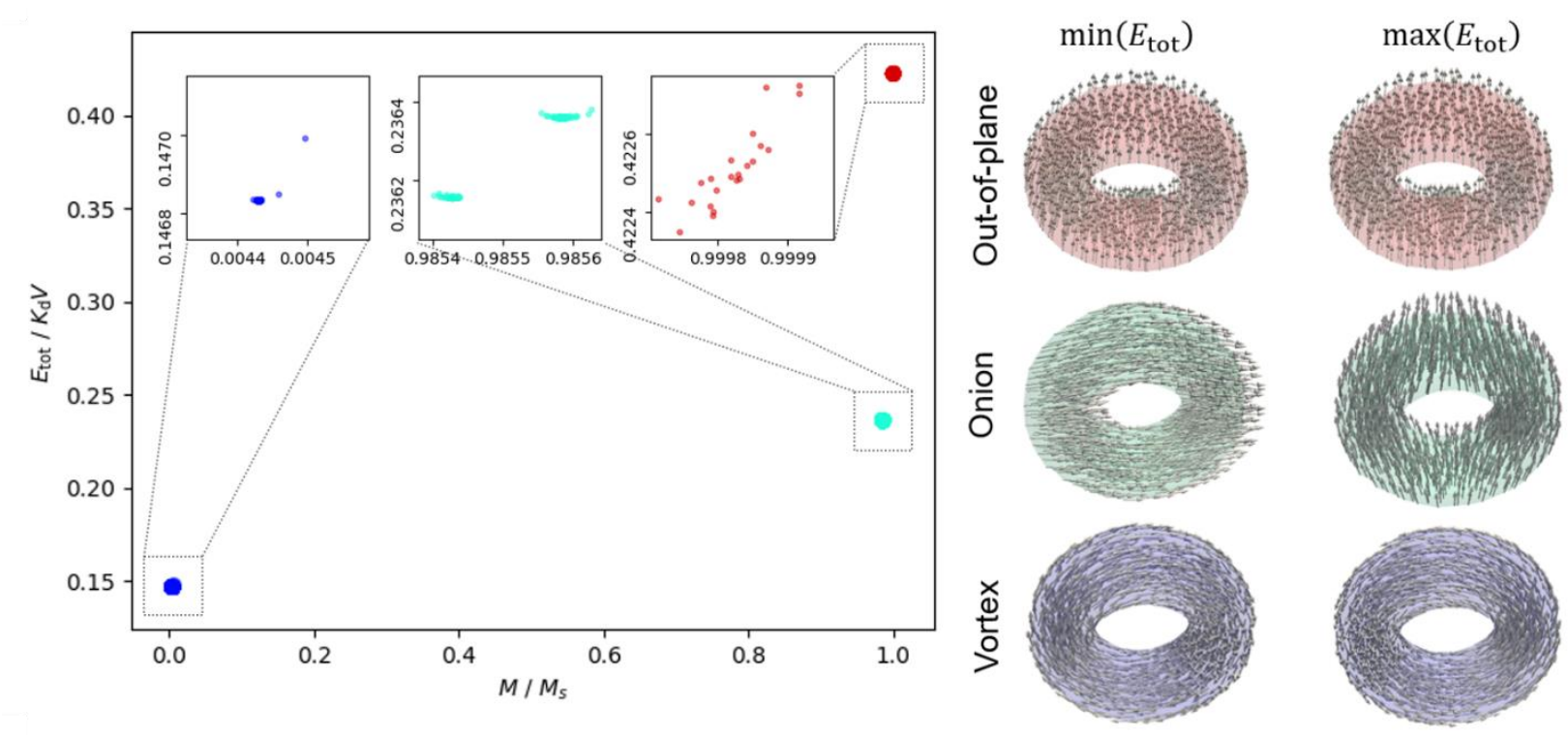

Figure S5 | Repeated random initializations. (Left) Micromagnetic remanent states resulting from 1000 random initializations, with insets at higher resolution, for a toroid with geometry $e=0.7, r / R=0.4$, and $d_{\mathrm{tot}}=70 \mathrm{~nm}$. (Right) Visualizations of the magnetic remanent states, with the highest and lowest energy states shown for each group.

The simulations produced 548 vortex states, 430 onion states, and 22 out-of-plane states. Small variations of the final energy were found within each state (on the order of $10^{-4} K_{\mathrm{d}} V$ ): for the vortex state, the difference between the highest and lowest $E_{\text {tot }}$ states was caused by a change in vortex direction; for the onion state, high and low energy states were perpendicular to one another; and for the out-of-plane states, no visible change was observed between high and low states - in this case small magnetization adjustments result in a relatively larger energy change since the state is much less stable than the other two.

These results illustrate how at this geometry, the vortex state is likely to be the true ground-state whilst onion and out-of-plane geometries represent valid local energy minima with significantly higher total energies.

c) Reconfiguration energy barrier

For a typical toroid geometry ( $e=0.7, r / R=0.4$, and $d_{\mathrm{tot}}=70 \mathrm{~nm}$ ), the minimum energy pathway was estimated using the nudged elastic band (NEB) method implemented in MERRILL ${ }^{18}$ for transitions from both uniform $\mathrm{z}$ and onion states to the vortex state (SI Figure S6). From our results, the uniform $z$ state is unstable, with a negligible energy barrier meaning that magnetic moments rotate smoothly into the vortex state. The transition from the onion state, however, has a sizable energy barrier of $\sim 0.43 \times 10^{-18} \mathrm{~J}$, which will be robust to thermal fluctuations at normal body temperature, and even to fluctuations on a paleomagnetic scale as shown by Muxworthy et al. ${ }^{19}$ From visualizations of the pathway, this barrier is associated with pushing of the domain wall clockwise around the ring and then removing the wall entirely. Converting particles out of the onion state and into the vortex state would therefore be more difficult but could be achieved by magnetizing them along $\mathrm{z}$ and allowing relaxation. 

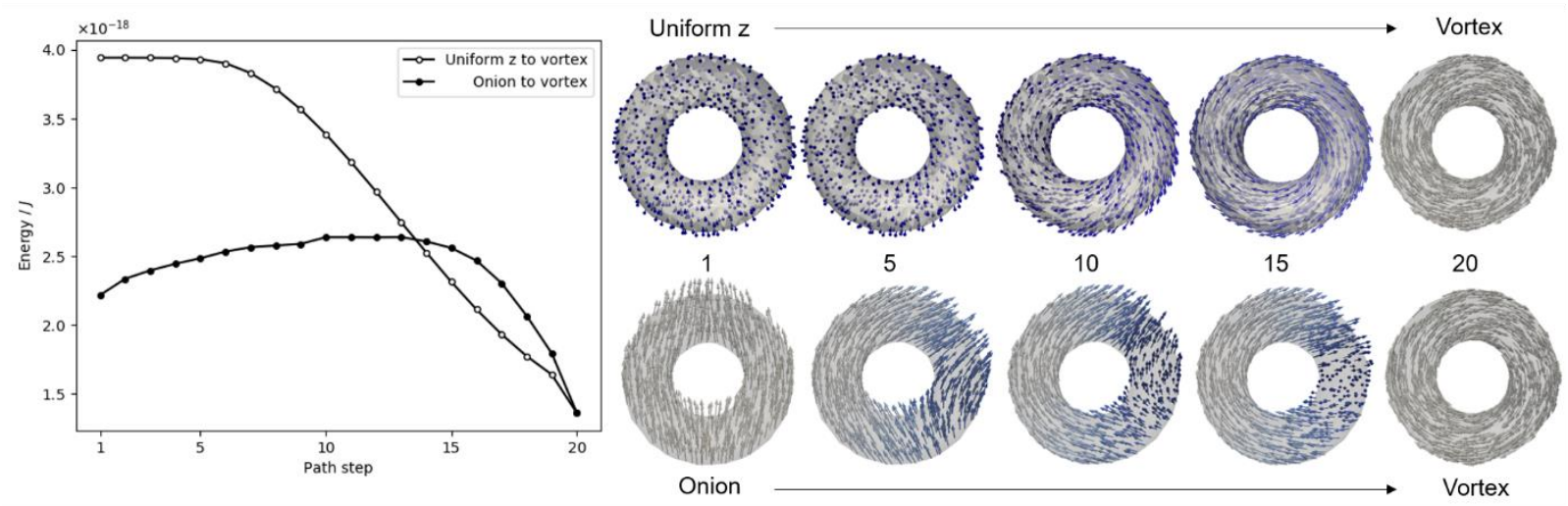

Figure S6 | Reconfiguration energy barrier. (Left) Minimum energy pathways calculated with 20 images between initial and final states for the transition of uniform $z$ to vortex and onion to vortex. (Right) Intermediate configurations visualized for each transition, where blue represents out-of-plane magnetization components.

\section{d) Initial magnetization}

Micromagnetic simulation results are influenced by the initial magnetization input. Several phase diagrams were calculated by initializing from uniform $x / y / z$ and from five different random magnetizations, with an overview of all simulations shown in SI Figure S7. Results with the lowest $E_{\text {tot }}$ were grouped and used to produce the lower bound "ground state" phase diagram (dashed lines in Figure 2) while those with the highest $M / M_{\mathrm{S}}$ were grouped to produce the upper bound "metastable" phase diagram (circles in Figure 2). 


$$
e=0.0
$$

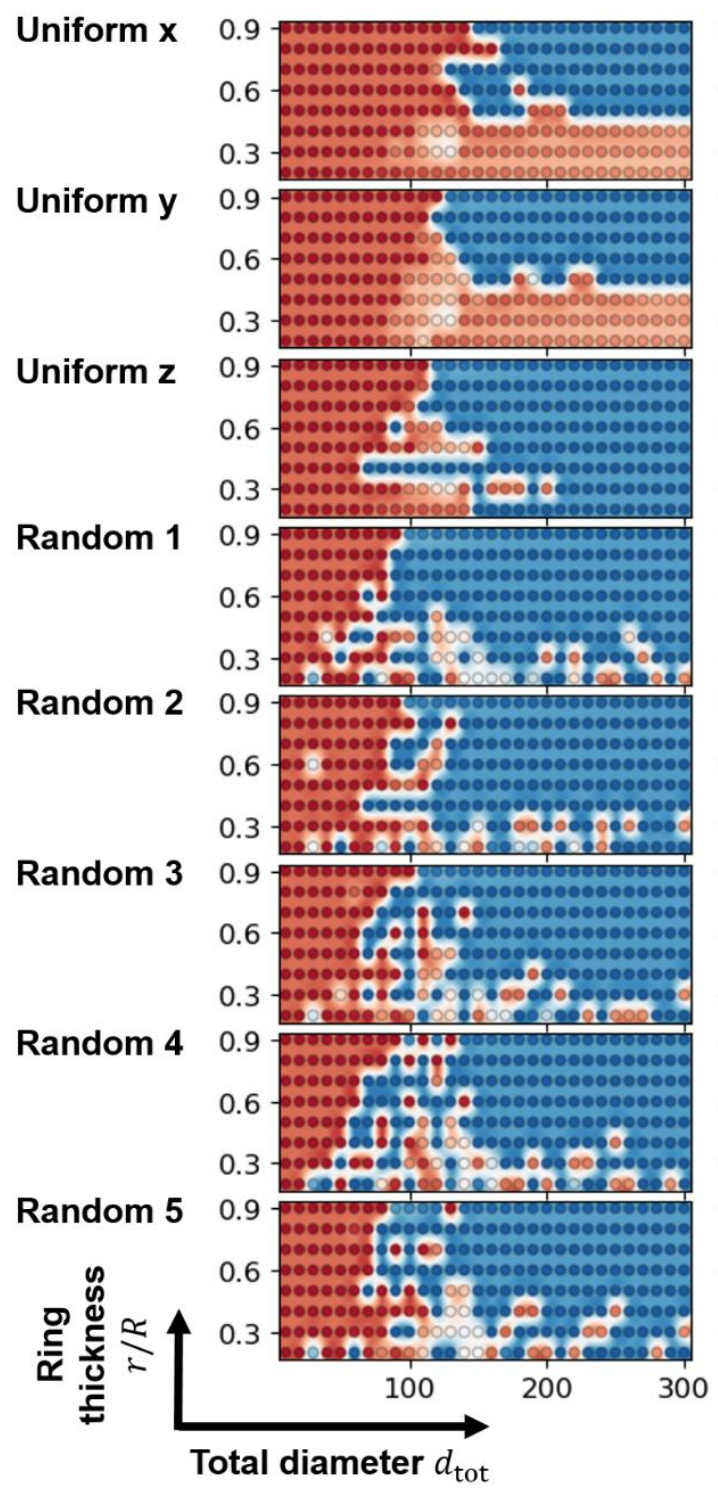

$$
e=0.7
$$
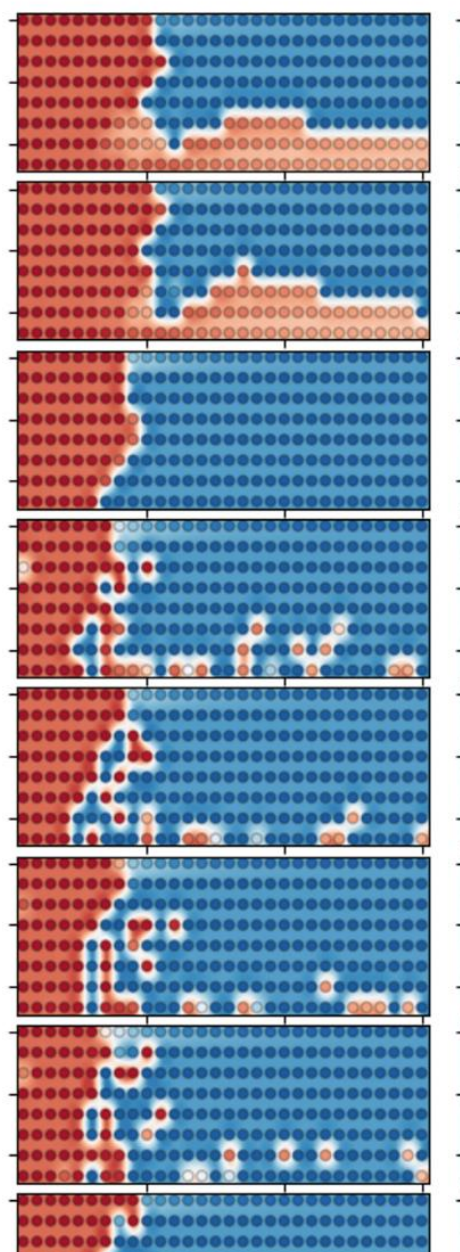

porovobe000000000000000000000
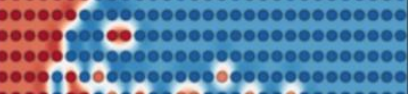

$100 \quad 200 \quad 300$
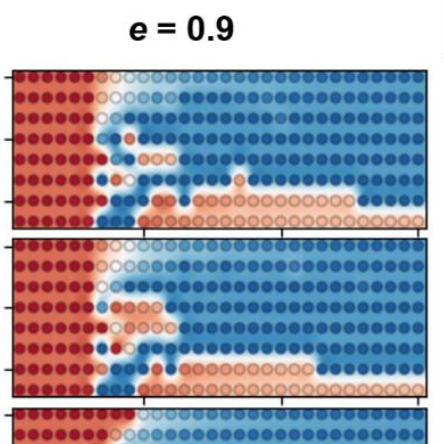

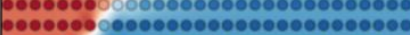

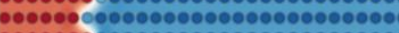
bov00000000000000000000000

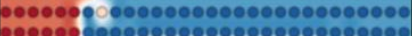
becovo

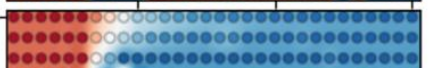

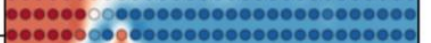
0.00090906000000000000000

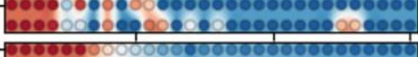

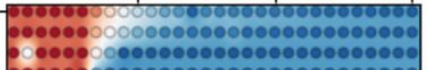
pover

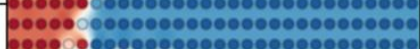

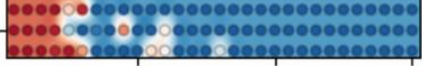
ए00000000000000000000000 6000000000000000000000000000 forever

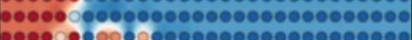

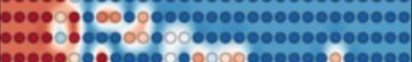

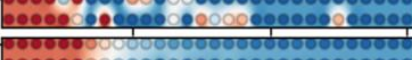

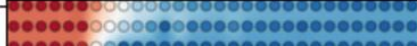
for

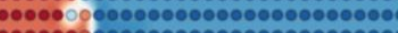

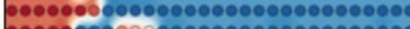

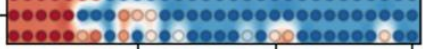
एक्षण000000000000000000000

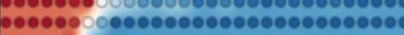
-

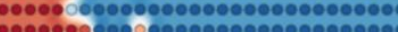
60000000000000000000000000 100 $100 \quad 200 \quad 300$

Figure S7 | Phase diagrams from different initial states. Phase diagrams are shown in the same style as Figure 2, with $x$ axis of $d_{\text {tot }}$ spanning 10 to $300 \mathrm{~nm}$ and $y$ axis of $r / R$ spanning 0.2 to 0.9 and points colored by the value of $M / M_{\mathrm{S}}$ for each diagram.

e) Twisted onion state

As reported in the main text, high $M / M_{\mathrm{S}}$ states in the region from $d_{\text {tot }}=70$ to $110 \mathrm{~nm}$ and $r / R=0.2$ to 0.4 adopt a previously unreported "twisted onion" state; similar to the onion state except that the $z$ magnetization component twists from up to down as the $x y$ component curls symmetrically around the particle (SI Figure S8). This is a consequence of shape anisotropy, whereby this configuration minimizes the area occupied by the magnetic poles of the particle. 

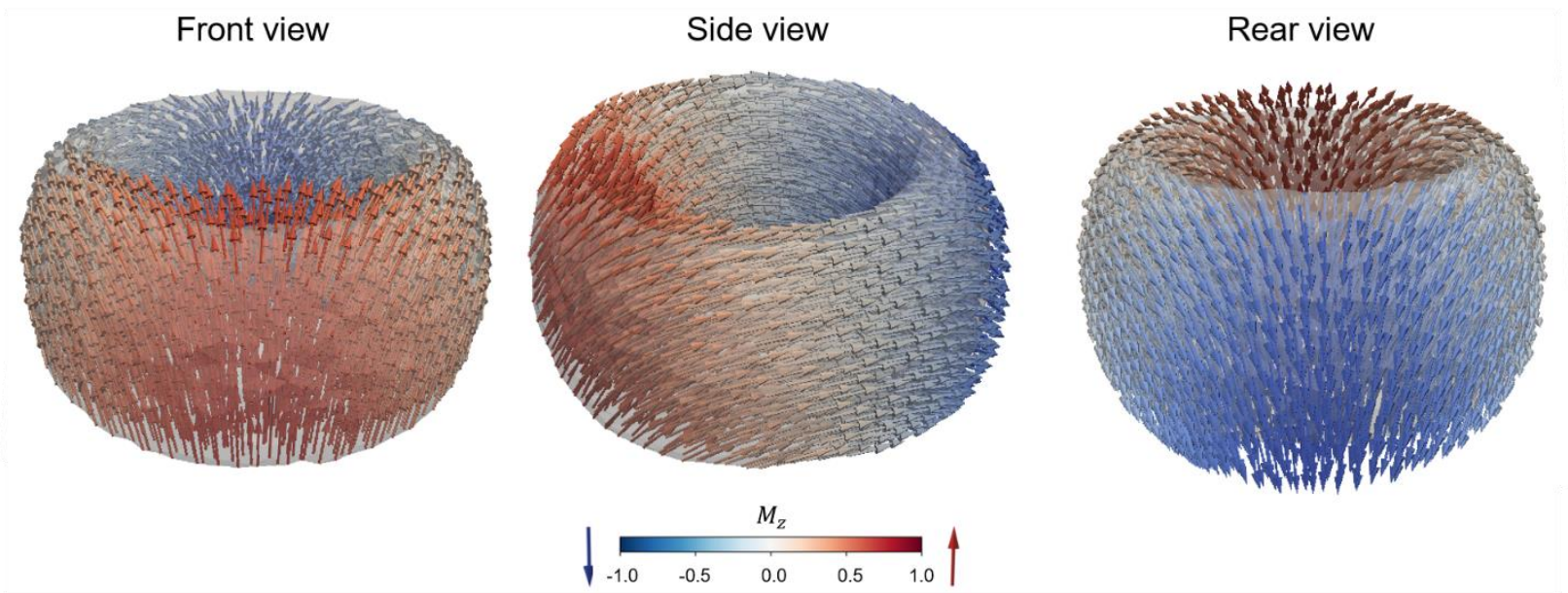

Figure S8 $\mid$ Twisted onion state. Visualization of the twisted onion remanent state for $e=0.9, r / R=0.3$, and $d_{\mathrm{tot}}=90 \mathrm{~nm}$ taken from initialization "Random 1", viewed from three angles with vectors colored by the $z$-component of magnetization.

\section{f) Size variation of extracted ring geometries}

For ring geometries extracted by tomography shown in Figure 3, the remanent states were calculated from uniform $x, y$, and $z$ initial states and net magnetization determined for each (SI Figure S9).

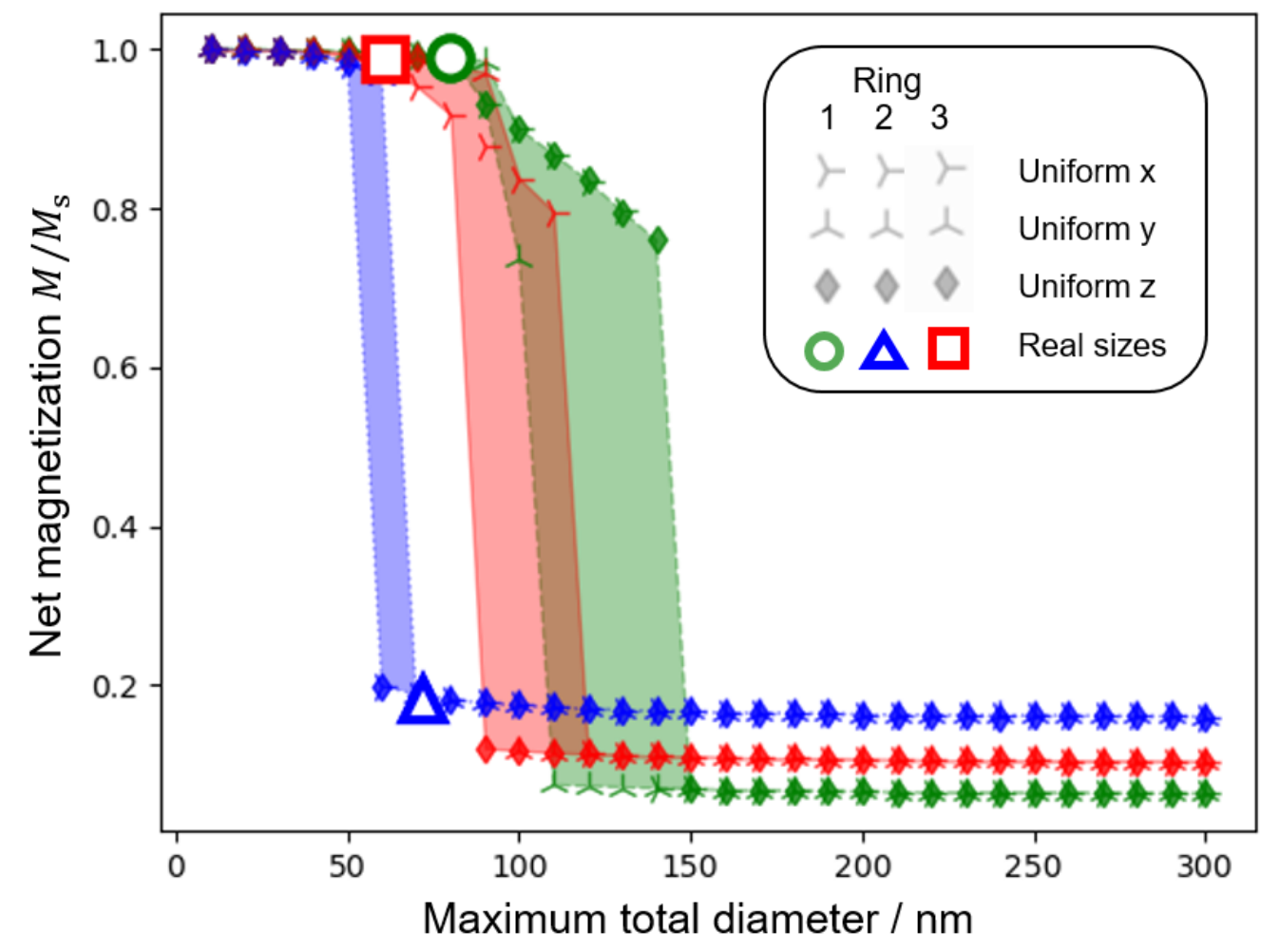

Figure S9 Size variation of extracted ring geometries. Variation in net magnetization of remanent states for the green, blue and red extracted ring geometries simulated at different sizes from uniform $x, y$, and $z$ initializations. Values obtained at the real size of the green, blue, and red particles are shown by the open circle, triangle, and square, respectively. Regions between maximum and minimum $M / M_{\mathrm{s}}$ at each size are colored and bounded by dashed, dotted, and solid lines for the green, blue, and red particles, respectively. 
g) Onion state/vortex state transition pathway for real geometries

The transition magnetization configurations obtained for the energy barrier calculations in Figure $5 \mathrm{c}$ illustrate the differences in switching mechanisms that can be introduced by various defects (SI Figure S10).
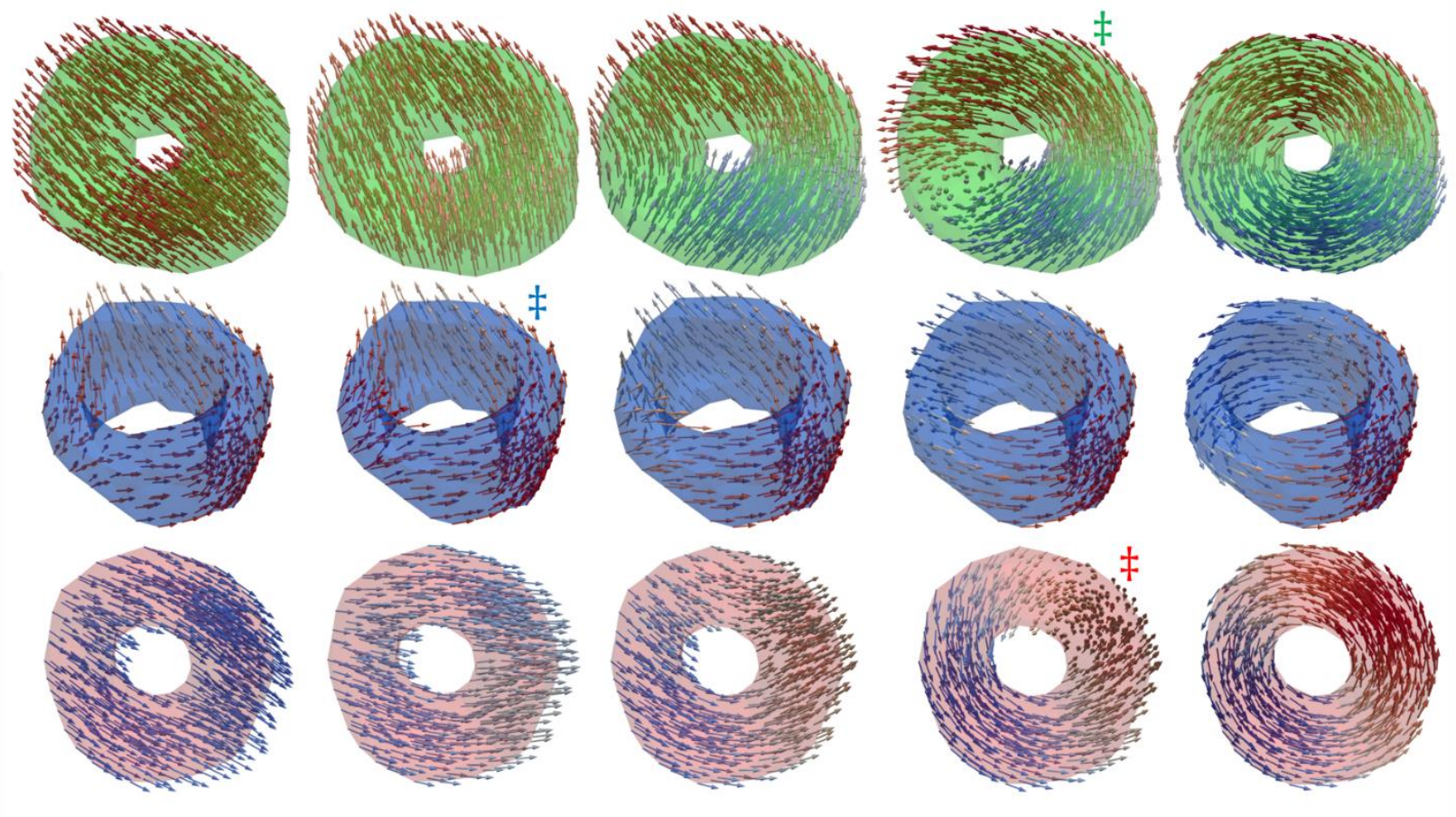

\section{Onion state}

\section{‡ Transition state}

Figure S10 | Onion state/vortex state transition for real geometries. The magnetization configurations of the green, blue, and red rings are shown for the onion state, vortex state, and three intermediate states calculated using an NEB algorithm. The highest energy (transition) states are marked by double daggers and arrows are colored red/blue depending on their alignment to the positive/negative $x$ axis direction.

\section{Extra details on methods}

a) Chemical synthesis

Nano-magnetites were synthesized using a hydrothermal method with iron(III) nitrate nonahydrate and sodium phosphate monobasic dehydrate precursors, heated to $220{ }^{\circ} \mathrm{C}$ for 48 hours. The particles were then washed with deionized water and dried at $60{ }^{\circ} \mathrm{C}$ for 6 hours to initially form nano-hematites. The nanohematites were subsequently extracted via carbon reduction to obtain nano-magnetite. ${ }^{20-22}$ Nanomaghemites were also obtained by oxidizing the magnetite.

b) TEM acquisition details

EDX data in Figure 1 were recorded using a Nion UltraSTEM, operating at $60 \mathrm{keV}$ in STEM mode. The tilt series used to recreate the tomography reconstruction was acquired using an FEI Tecnai Osiris instrument operating in HAADF-STEM mode. 46 images were recorded at $3^{\circ}$ intervals from $-68^{\circ}$ and $+70^{\circ}$. The post-acquisition micrograph in Figure $3 \mathrm{~b}$ was recorded using an FEI Tecnai F20 instrument with a 200 $\mathrm{keV}$ field emission gun (FEG) operating in bright field mode. 


\section{c) Tomographic reconstruction details}

Images were first aligned using a phase cross-correlation method on Sobel-filtered data. The compressed sensing reconstruction was formulated as in Goris et al. ${ }^{23}$ with the weight on the data-term of $\mu=10$. This was implemented using 200 iterations of a Chambolle-Pock algorithm. ${ }^{24}$ At this point the change between successive iterations was of order $10^{-5}$, which indicates that the reconstruction converged to a stable solution. Commercial software Avizo was then used for thresholding particles and extracting isosurfaces.

d) Meshing details

Isosurfaces for tomography-extracted shapes were generated as described in 6.c) above, and isosurfaces for ideal toroids were created in Blender ${ }^{25}$ using the mathematical formulae discussed in Section 4. The isosurfaces for intermediate interpolated shapes were also generated in Blender using a shrinkwrap modifier.

As described in the main text, tetrahedral volume meshes were created for each geometry, nodes were spaced such that when the mesh was rescaled to the largest diameter $(300 \mathrm{~nm})$ the maximum spacing would be $<9 \mathrm{~nm}$, corresponding approximately to the exchange length of magnetite. This is important for allowing the simulation to correctly resolve magnetization within the mesh geometry. The same mesh for each $e$ and $r / R$ combination was scaled down to simulate lower diameter particles.

e) Micromagnetic simulation details

Our simulations account for energy contributions from exchange coupling, cubic crystalline anisotropy, and demagnetization energy terms and were run with zero applied field, at $20{ }^{\circ} \mathrm{C}$, specifying the [111] crystal axis along the particle $\mathrm{z}$ axis in accordance with experimental results. ${ }^{5}$ Each simulation was run either until a stable energy solution was reached or for 10,000 iterations, saving the final magnetization configuration and micromagnetic energies in units of $K_{\mathrm{d}} V$ (which is the stray field energy density multiplied by the relevant volume).

The magnetic parameters used for magnetite at $20{ }^{\circ} \mathrm{C}$ were calculated by MERRILL to be: exchange coupling constant, $1.335 \times 10^{-11} \mathrm{~J} \mathrm{~m}^{-1}$; crystalline anisotropy constant $K_{1},-1.327 \times 10^{4} \mathrm{~J} \mathrm{~m}^{-3}$; and saturation magnetization, $4.808 \times 10^{5} \mathrm{~A} \mathrm{~m}^{-1}$. Higher order crystalline anisotropy terms were ignored. $3 \mathrm{D}$ visualizations of magnetization were created using ParaView. ${ }^{26}$

f) Magnetic stray field calculations

Magnetic stray field calculations in Figure 5b were created by first linearly interpolating magnetization data from a tetrahedral mesh onto a regular 3D grid. This resulted in non-zero magnetization in the central hole of the toroids due to its inclusion within the convex hull of the toroid, and so a kd-tree construction was used to find the nearest neighbors between the interpolated and tetrahedral-mesh datasets; this was subsequently used to apply a mask to retain only points interpolated within the original particle volume.

The 3D gridded magnetization was then projected into $2 \mathrm{D}$ to obtain the projected magnetization $\boldsymbol{M}_{\perp}$ using a modified version of the Joseph pixel projection algorithm. ${ }^{27}$. From this the magnetic phase shift associated with this magnetization and hence the projected $B$-field was calculated using the method described by Beleggia and Zhu. ${ }^{28}$ Following their method the magnetic phase was calculated from the projected magnetization using the formula given by Beleggia and $\mathrm{Zhu}^{28}$

$$
\tilde{\phi}_{\mathrm{m}}\left(k_{\mathrm{x}}, k_{\mathrm{y}}\right)=\frac{i e \mu_{0}}{h} \frac{\left[\widetilde{\boldsymbol{M}}_{\perp}\left(k_{\mathrm{x}}, k_{\mathrm{y}}\right) \times \boldsymbol{k}_{\perp}\right]}{k_{\perp}^{2}},
$$

[Equation S3]

where the tilde denotes a 2D Fourier transform, $k_{\mathrm{x}}, k_{\mathrm{y}}$ are spatial frequencies, $h$ is the Planck constant, $\mu_{0}$ is the vacuum permeability, and $\boldsymbol{k}_{\perp}=\left(k_{\mathrm{x}}, k_{\mathrm{y}}, 0\right)$. 
Finally, this allows calculation of the projected $B$-field from the electron phase shift by

$$
\boldsymbol{B}_{\perp}(x, y)=\frac{\hbar}{e}\left(\begin{array}{c}
-\partial / \partial_{y} \\
\partial / \partial_{x}
\end{array}\right) \phi_{\mathrm{m}}
$$

[Equation S4]

Note that throughout this work the projected B-field component $j$ is defined by

$$
B_{j}^{p}(x, y)=\int_{-\infty}^{\infty} B_{j}(x, y, z) d z
$$

[Equation S5]

as described by Kasama et al., ${ }^{29}$ and hence the projected B field is simply $\boldsymbol{B}_{\perp}=B_{x}^{p} \hat{\boldsymbol{\imath}}+B_{y}^{p} \widehat{\boldsymbol{k}}$.

This projected B-field was subsequently calculated for a $300 \mathrm{~nm} \times 300 \mathrm{~nm}$ area centered on the particle. The internal B-field was then masked out and an average value for the stray field was calculated for the remaining pixels.

\section{References}

(1) Ali, A.; Zafar, H.; Zia, M.; Ul Haq, I.; Phull, A. R.; Ali, J. S.; Hussain, A. Synthesis, Characterization, Applications, and Challenges of Iron Oxide Nanoparticles. Nanotechnol. Sci. Appl. 2016, 9, 49-67. https://doi.org/10.2147/NSA.S99986.

(2) Cornell, R. M.; Schwertmann, U. The Iron Oxides: Structure, Properties, Reactions, Occurences and Uses, 2nd ed.; Wiley-VCH Verlag GmbH \& Co. KGaA: Weinheim, 2003. https://doi.org/10.1002/3527602097.

(3) Laurent, S.; Forge, D.; Port, M.; Roch, A.; Robic, C.; Vander Elst, L.; Muller, R. N. Magnetic Iron Oxide Nanoparticles: Synthesis, Stabilization, Vectorization, Physicochemical Characterizations, and Biological Applications. Chem. Rev. 2008, 108 (6), 2064-2110. https://doi.org/10.1021/cr068445e.

(4) Das, R.; Witanachchi, C.; Nemati, Z.; Kalappattil, V.; Rodrigo, I.; García, J. Á.; Garaio, E.; Alonso, J.; Lam, V. D.; Le, A.-T.; et al. Magnetic Vortex and Hyperthermia Suppression in Multigrain Iron Oxide Nanorings. Appl. Sci. 2020, 10 (3), 787. https://doi.org/10.3390/app10030787.

(5) Jia, C.-J.; Sun, L.-D.; Luo, F.; Han, X.-D.; Heyderman, L. J.; Yan, Z.-G.; Yan, C.-H.; Zheng, K.; Zhang, Z.; Takano, M.; et al. Large-Scale Synthesis of Single-Crystalline Iron Oxide Magnetic Nanorings. J. Am. Chem. Soc. 2008, 130 (50), 16968-16977. https://doi.org/10.1021/ja805152t.

(6) Tobin, A. G.; Paul, D. I. Stability of Ferromagnetic Domain Structures at Grain Boundaries. J. Appl. Phys. 1969, 40, 3611. https://doi.org/10.1063/1.1658244.

(7) Pike, C. R.; Roberts, A. P.; Verosub, K. L. Characterizing Interactions in Fine Magnetic Particle Systems Using First Order Reversal Curves. J. Appl. Phys. 1999, 85 (9), 6660-6667. https://doi.org/10.1063/1.370176.

(8) Roberts, A. P.; Pike, C. R.; Verosub, K. L. First-Order Reversal Curve Diagrams: A New Tool for Characterizing the Magnetic Properties of Natural Samples. J. Geophys. Res. Solid Earth 2000, 105 (B12), 28461-28475. https://doi.org/10.1029/2000JB900326.

(9) Zhao, X.; Roberts, A. P.; Heslop, D.; Paterson, G. A.; Li, Y.; Li, J. Magnetic Domain State Diagnosis Using Hysteresis Reversal Curves. J. Geophys. Res. Solid Earth 2017, 122 (7), 4767-4789. https://doi.org/10.1002/2016JB013683.

(10) Roberts, A. P.; Almeida, T. P.; Church, N. S.; Harrison, R. J.; Heslop, D.; Li, Y.; Li, J.; Muxworthy, A. R.; Williams, W.; Zhao, X. Resolving the Origin of Pseudo-Single Domain Magnetic Behavior. 
J. Geophys. Res. Solid Earth 2017, 122 (12), 9534-9558. https://doi.org/10.1002/2017JB014860.

(11) Harrison, R. J.; Zhao, X.; Hu, P.; Sato, T.; Heslop, D.; Muxworthy, A. R.; Oda, H.; Kuppili, V. S. C.; Roberts, A. P. Simulation of Remanent, Transient, and Induced FORC Diagrams for Interacting Particles With Uniaxial, Cubic, and Hexagonal Anisotropy. J. Geophys. Res. Solid Earth 2019, 124 (12), 12404-12429. https://doi.org/10.1029/2019JB018050.

(12) Rave, W.; Ramstöck, K.; Hubert, A. Corners and Nucleation in Micromagnetics. J. Magn. Magn. Mater. 1998, 183 (3), 329-333. https://doi.org/10.1016/S0304-8853(97)01086-X.

(13) Schabes, M. E.; Bertram, H. N. Magnetization Processes in Ferromagnetic Cubes. J. Appl. Phys. 1988, 64, 1357. https://doi.org/10.1063/1.341858.

(14) Yang, Y.; Liu, X.-L.; Yi, J.-B.; Yang, Y.; Fan, H.-M.; Ding, J. Stable Vortex Magnetite Nanorings Colloid: Micromagnetic Simulation and Experimental Demonstration. J. Appl. Phys 2012, 111, 044303. https://doi.org/10.1063/1.3684963.

(15) Scholz, W.; Guslienko, K. Y.; Novosad, V.; Suess, D.; Schrefl, T.; Chantrell, R. W.; Fidler, J. Transition from Single-Domain to Vortex State in Soft Magnetic Cylindrical Nanodots. J. Magn. Magn. Mater. 2003, 266, 155-163. https://doi.org/10.1016/S0304-8853(03)00466-9.

(16) Gregurec, D.; Senko, A. W.; Chuvilin, A.; Reddy, P. D.; Sankararaman, A.; Rosenfeld, D.; Chiang, P.-H.; Garcia, F.; Tafel, I.; Varnavides, G.; et al. Magnetic Vortex Nanodiscs Enable Remote Magnetomechanical Neural Stimulation. ACS Nano 2020, $14,8045$. https://doi.org/10.1021/acsnano.0c00562.

(17) Nowak, U. Thermally Activated Reversal in Magnetic Nanostructures. In Annual Reviews Of Computational Physics IX; Stauffer, D., Ed.; World Scientific: Singapore, 2001; pp 105-152. https://doi.org/10.1142/4625.

(18) Conbhui, P. O.; Williams, W.; Fabian, K.; Ridley, P.; Nagy, L.; Muxworthy, A. R. MERRILL: Micromagnetic Earth Related Robust Interpreted Language Laboratory. Geochemistry, Geophys. Geosystems 2018, 19, 1080-1106. https://doi.org/10.1002/2017GC007279.

(19) Muxworthy, A. R.; Dunlop, D. J.; Williams, W. High-Temperature Magnetic Stability of Small Magnetite Particles. J. Geophys. Res. Solid Earth 2003, 108 (B5). https://doi.org/10.1029/2002jb002195.

(20) Islam, M. S.; Kusumoto, Y.; Abdulla-Al-Mamun, M. Novel Rose-Type Magnetic (Fe3O4, $\gamma$-Fe2O3 and $\alpha$-Fe2O3) Nanoplates Synthesized by Simple Hydrothermal Decomposition. Mater. Lett. 2012, 66, 165-167. https://doi.org/10.1016/j.matlet.2011.08.057.

(21) Chen, Y. H.; Lin, C. C. Effect of Nano-Hematite Morphology on Photocatalytic Activity. Phys. Chem. Miner. 2014, 41, 727-736. https://doi.org/10.1007/s00269-014-0686-9.

(22) Hu, P.; Zhang, S.; Wang, H.; Pan, D.; Tian, J.; Tang, Z.; Volinsky, A. A. Heat Treatment Effects on Fe3O4 Nanoparticles Structure and Magnetic Properties Prepared by Carbothermal Reduction. J. Alloys Compd. 2011, 509, 2316-2319. https://doi.org/10.1016/j.jallcom.2010.10.211.

(23) Goris, B.; Van den Broek, W.; Batenburg, K. J.; Heidari Mezerji, H.; Bals, S. Electron Tomography Based on a Total Variation Minimization Reconstruction Technique. Ultramicroscopy 2012, 113, 120-130. https://doi.org/10.1016/J.ULTRAMIC.2011.11.004.

(24) Chambolle, A.; Pock, T. A First-Order Primal-Dual Algorithm for Convex Problems with Applications to Imaging. J. Math. Imaging Vis. 2011, 40 (1), 120-145. https://doi.org/10.1007/s10851-010-0251-1. 
(25) Blender 2.80 - Open source 3D creation suite https://www.blender.org/.

(26) Ahrens, J.; Geveci, B.; Law, C. ParaView: An End-User Tool for Large Data Visualization; Elsevier, 2005.

(27) Joseph, P. M. An Improved Algorithm for Reprojecting Rays through Pixel Images. IEEE Trans. Med. Imaging 1982, I (3), 192-196. https://doi.org/10.1109/TMI.1982.4307572.

(28) Beleggia, M.; Zhu, Y. Electron-Optical Phase Shift of Magnetic Nanoparticles I. Basic Concepts. Philos. Mag. 2003, 83 (8), 1045-1057. https://doi.org/10.1080/0141861031000066166.

(29) Kasama, T.; Dunin-Borkowski., R. E.; Beleggia, M. Electron Holography of Magnetic Materials. In Holography - Different Fields of Application; Monroy, F., Ed.; InTech, 2011; pp 53-80. https://doi.org/10.5772/22366. 